Mol. Cryst. Liq. Cryst., 1983, Vol. 98, pp. 183-191

$0026-8941 / 83 / 9804-0183 / \$ 18.50 / 0$

(C) 1983 Gordon and Breach, Science Publishers, Inc.

Printed in the United States of America

\title{
Electrohydrodynamic Behavior in Twisted-Wedge Nematic Structures ${ }^{\dagger}$
}

\author{
J.A. MARTIN-PEREDA, M. A. MURIEL and F. J. LOPEZ \\ Departamento de Electronica Cuantica, E. T. S. Ing. Telecomunicacion, \\ Universidad Politecnica de Madrid, Ciudad Universitaria, Madrid-3, Spain
}

(Received Jamuary 31, 1983)

A new type of domain for nematic liquid crystals with a twisted-wedge structure is presented. This new type of domain appears from the low frequency range to $10 \mathrm{kHz}$. This behavior was observed for square and pulsed excitations.

The liquid crystal was $N$-( $p$-methoxybenzylidene $)-p^{\prime}-$ butylaniline) (MBBA) used at room temperature. These domains offer a higher degree of complexity than conventional Williams domains. The corresponding stability chart is presented.

\section{INTRODUCTION}

In nematics, electrohydrodynamic instabilities have been extensively studied by numerous workers and seem presently to be well understood, at least at low frequencies. Different types of domain appear depending on the voltage and frequency applied to the nematic liquid crystal cell. Two regions clearly appear, separated by a cut-off frequency, $f_{c}$. For dc or ac fields where $f<f_{c}$, there are two different modes, Williams domains (striations or cellular patterns) and dynamic scattering, both associated with hydrodynamic flow. For ac fields with $f>f_{c}$, a fast turn-off mode appears with striations or chevrons with a few $\mu$ m-periodicity, associated with bend oscillations. For instance, in the case of MBBA, the plot of voltage threshold, $V_{c}$, of instabilities vs frequency $f$, of the applied field, has an $S$-shape, much more pronounced for square wave excitation than for sine wave

${ }^{\dagger}$ Presented at the Ninth International Liquid Crystal Conference, Bangalore, December 6-10, 1982. 
excitation; for a $50 \mu \mathrm{m}$-thick sample, $f_{c}$ is between 75 and $100 \mathrm{~Hz}$. Voltages lie between 10 volts for the conduction regime (low frequencies) and around 100 volts for the dielectric regime (high frequencies). The sample was in almost every case contained in a sandwich cell having $\mathrm{SnO}_{2}$-coated glass plates, and the nematic liquid crystal had a homogeneous planar texture. ${ }^{1,2,3}$ Williams domains are at angles to the direction of rubbing. Recently, a new type of domain, with a static aspect, was observed at frequencies above the disappearance of the chevron pattern. ${ }^{5}$ This structure appears in the dielectric region (till to $8 \mathrm{kHz}$ ) by raising the frequency at constant voltage until disappearance of the chevron structure. This new type of domain was observed both for homogeneous cells and for twisted geometries.

In this paper we extend some results previously reported by us ${ }^{6}$ showing a new stability chart with some features not presented before for nematic liquid crystal cells with twisted wedge geometries.

\section{EXPERIMENTAL}

The liquid crystal device employed in this work is similar to the one reported by us as a light deflector, both analogue ${ }^{7}$ and digital, ${ }^{8}$ and as an optical modulator. ${ }^{9}$ We have used the same wedge structure, with a prismatic angle around $2^{\circ}$ and a Mylar spacer in just one side of the cell. Internal transparent electrodes were deposited by the conventional spraying method. We have used $\mathrm{SnO}_{2}$ and ITO electrodes. However a different nematic liquid crystal and molecular configuration have been used in this case, namely MBBA in a twisted configuration in the wedge cell. The working temperature was room temperature and the MBBA had a large number of charge carriers. As is known, MBBA has a refractive index $\Delta n$ of about 0.24 and a negative dielectric anisotropy of $-0.53 \varepsilon_{0}$. The cell is shown in Figure 1.

With this configuration we have observed the behavior of the liquid crystal for frequencies between $0 \mathrm{~Hz}$ and $10 \mathrm{kHz}$, for different conditions of the applied electric field. We used square and pulsed signals with peak values up to 60 volts.

The diffraction pattern was observed by illuminating the sample with a parallel nonpolarized $0.5 \mathrm{~mW} \mathrm{He}-\mathrm{Ne}$ laser beam, normal to the front surface. With a lens, it was possible to see the domains inside the cell projected on a screen. With this method, by applying a dc voltage to the cell, one observes domain formation at 10 volts, as has been reported previously for non-twisted structures. ${ }^{10}$ However, the main features appear for ac voltages. The experimental set-up is shown in Figure 2. 


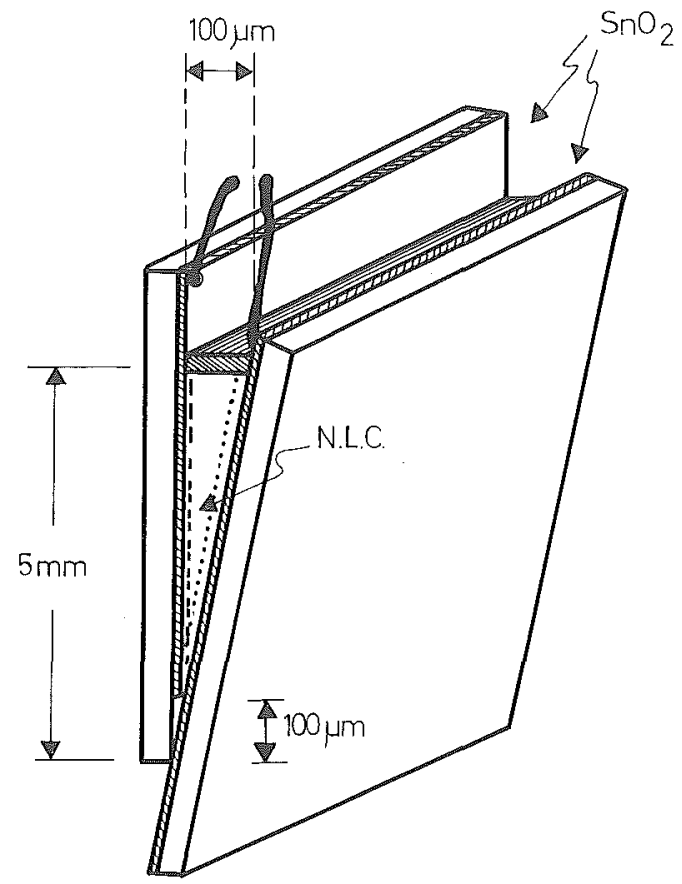

FIGURE 1 Liquid crystal wedge cell.

\section{RESULTS}

We have applied a square voltage, with zero volts as mean value, with frequencies from $1 \mathrm{~Hz}$ to $10 \mathrm{kHz}$ and found four different regions for every observed frequency. From 0 volts to a certain $V_{1}$, no diffraction pattern is obtained. From this $V_{1}$ to another $\dot{V}_{2}$, a structure similar to Williams domains can be observed. ${ }^{1,4}$ The size of these domains was the same for the entire range of frequencies. From $V_{2}$ to a certain value $V_{3}$ we got a very different diffraction pattern shown in Figure 3. As can be seen, this maintains the main features of the Williams domain diffraction pattern, but with two sets of points parallel to it. The corresponding domains are shown in Figure 4 . These domains are very different from the conventional Williams domains. The pattern is stationary at fixed voltage without any turbulence up to $V_{3}$, where a structure like the one shown in Figure 5 appears. This structure is very difficult to keep static and for still higher voltages turbulence dominates. The diffraction pattern is shown in Figure 6. Samples of high quality can show as many as 11 parallel lines comprising more than 31 points in each one. 


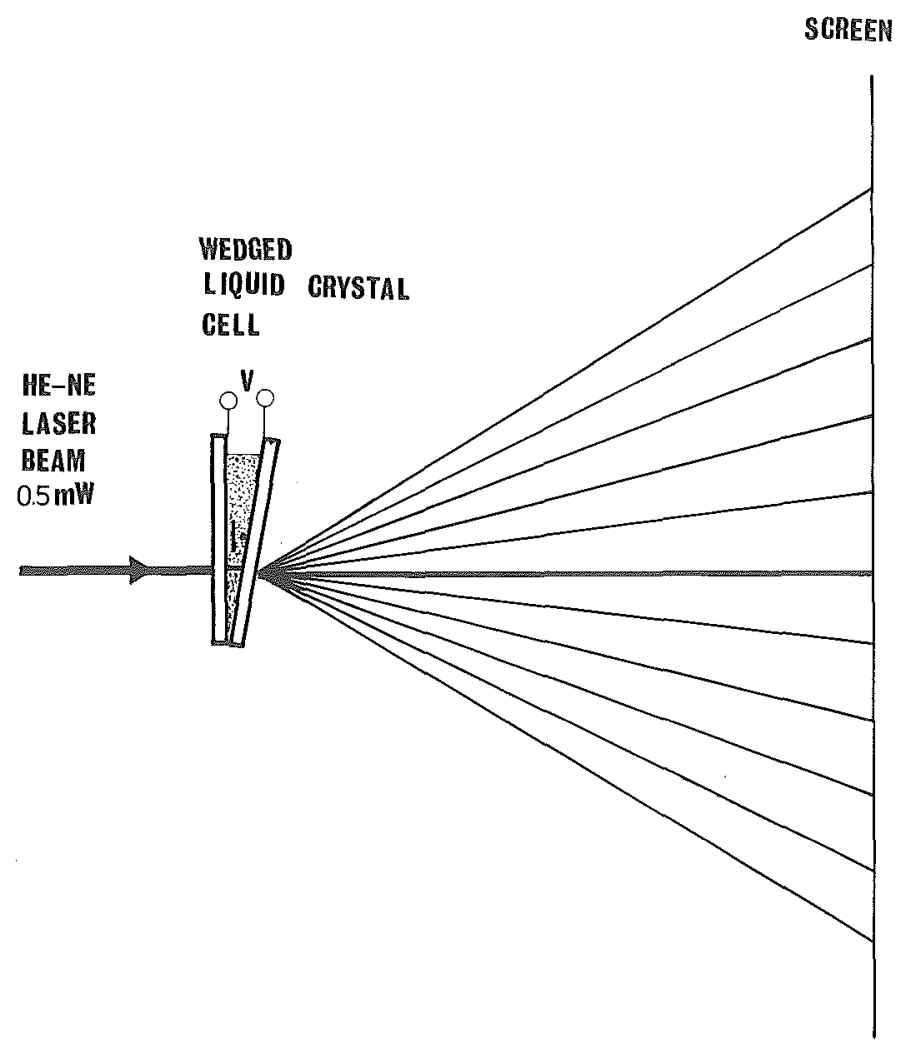

FIGURE 2 Experimental set-up.

The corresponding diagram for the above situation is shown in Figure 7 where the four regions indicated above are depicted. The main feature of this representation is the peak around $10 \mathrm{~Hz}$. The other interesting point is the strong increase in voltage from $1000 \mathrm{~Hz}$. The region below $10 \mathrm{~Hz}$ corresponds to electrohydrodynamic instabilities modulated at the same frequency as the voltage. This modulation can be seen clearly on the diffraction pattern. From $10 \mathrm{~Hz}$ the modulation disappears and the domains are static in nature. From $1000 \mathrm{~Hz}$, the voltage difference between different regions becomes smaller and becomes very close around $10 \mathrm{kHz}$. For higher frequencies, we were not able to obtain any diffraction pattern.

The results were very similar with a pulsed voltage. The corresponding stability chart is shown in Figure 8. The same peak at $10 \mathrm{~Hz}$ appears as does the strong increase in voltage from $1000 \mathrm{~Hz}$. It is interesting to point 


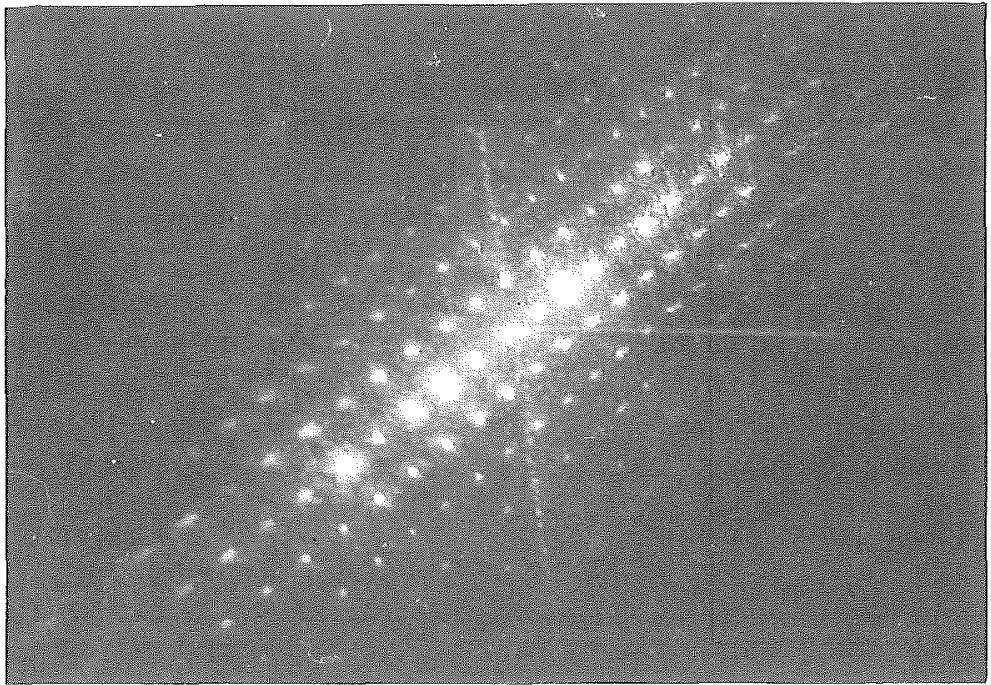

FIGURE 3 Diffraction pattern corresponding to voltages $V_{2}<V<V_{3}$ where $V_{2}$ and $V_{3}$ are defined in the text.

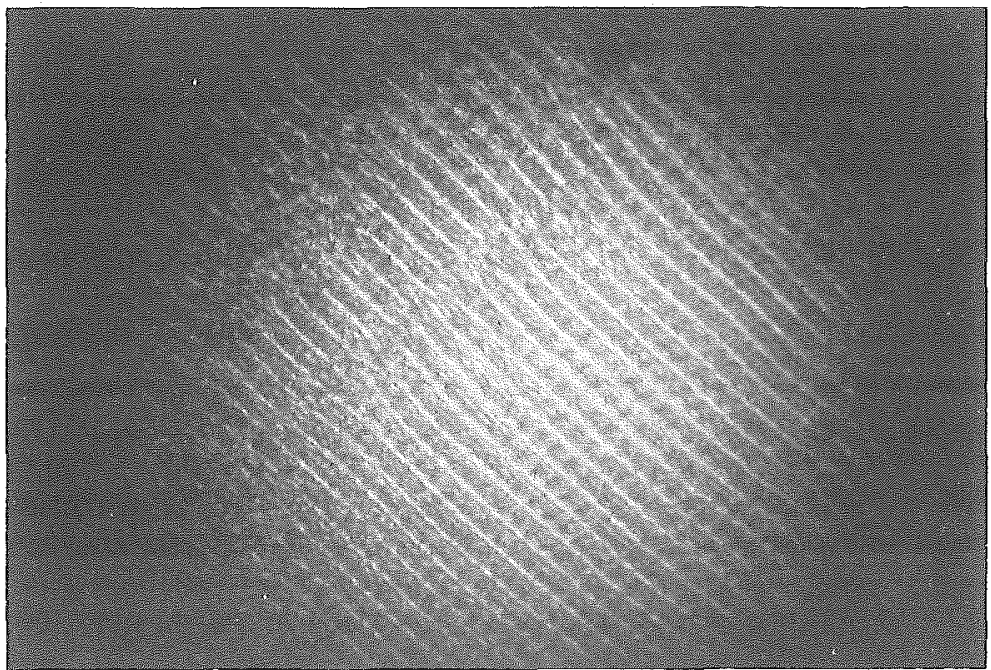

FIGURE 4 Domains corresponding to the diffraction pattern of Fig. 3. 


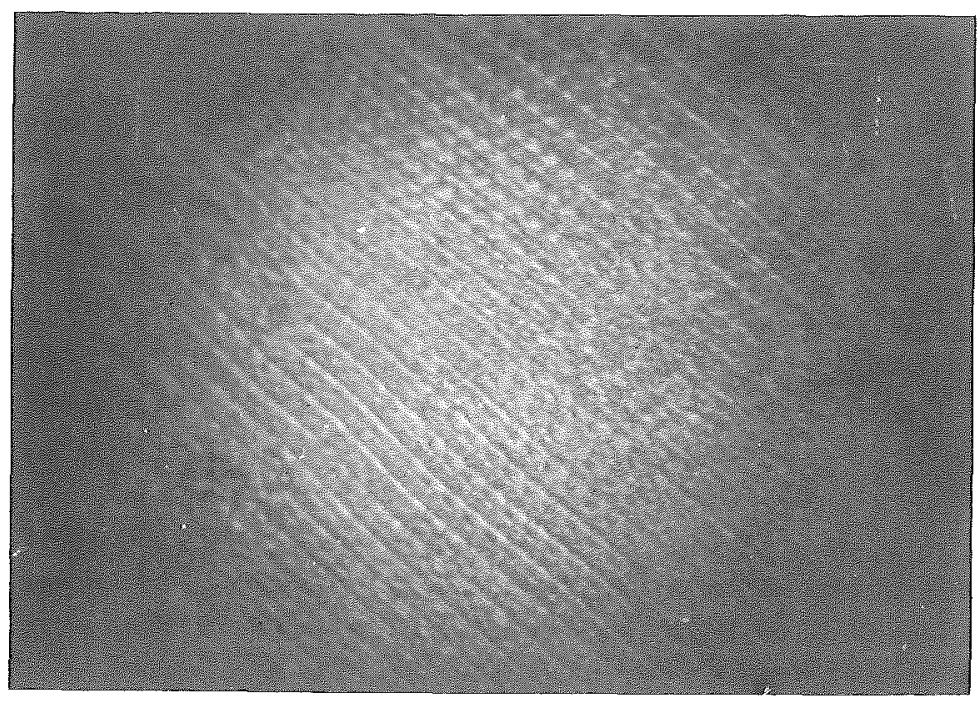

FIGURE 5 Domains corresponding to voltages near $V_{3}$.

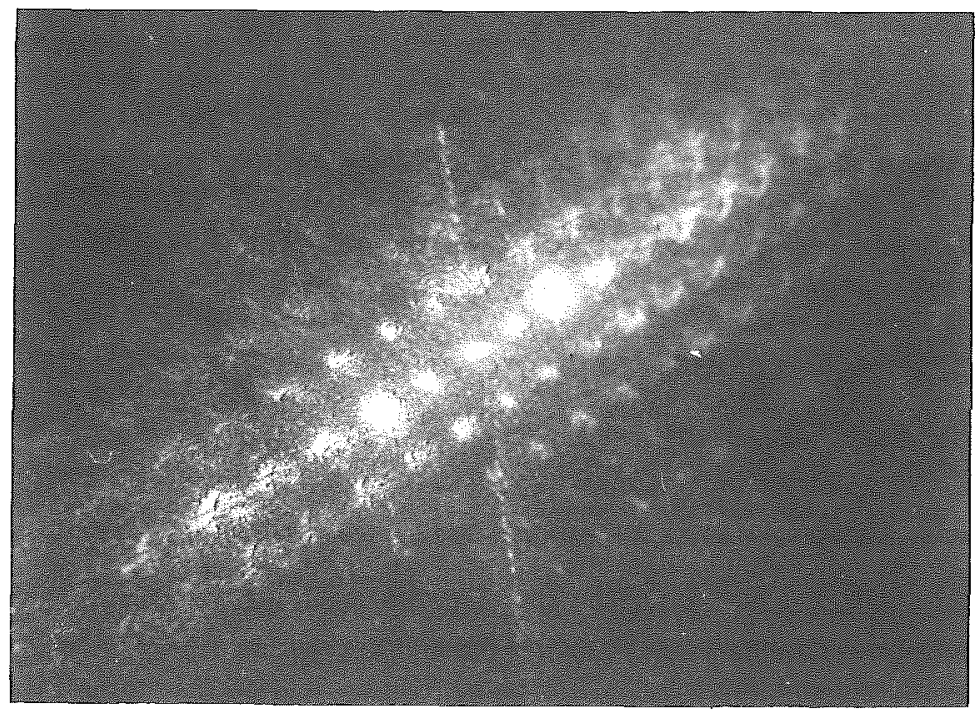

FIGURE 6 Diffraction pattern corresponding to voltages near $V_{3}$. 


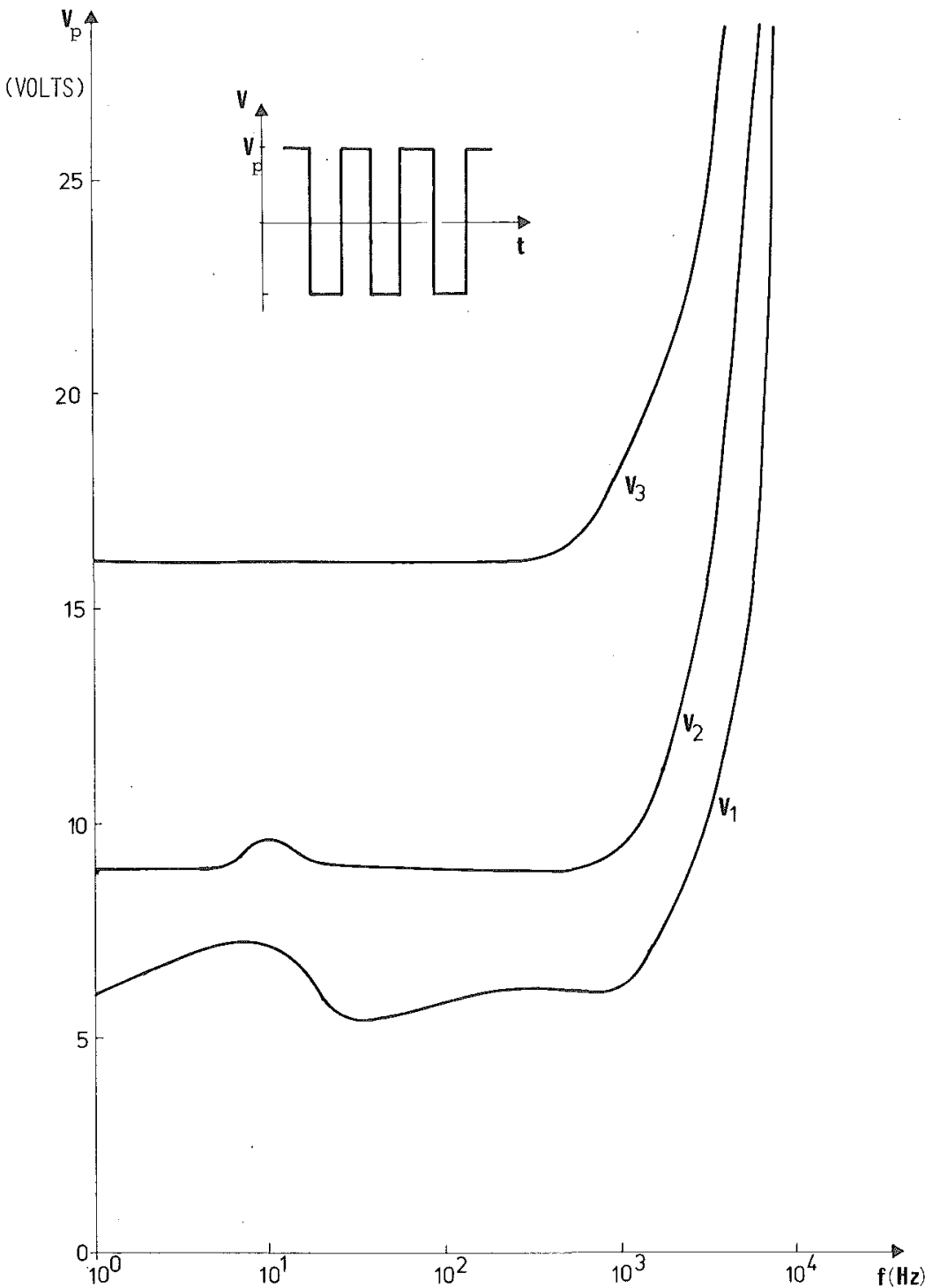

FIGURE 7 Stability chart of threshold voltage vs frequency in a twisted-wedge structure. The sample was excited with square waves. 


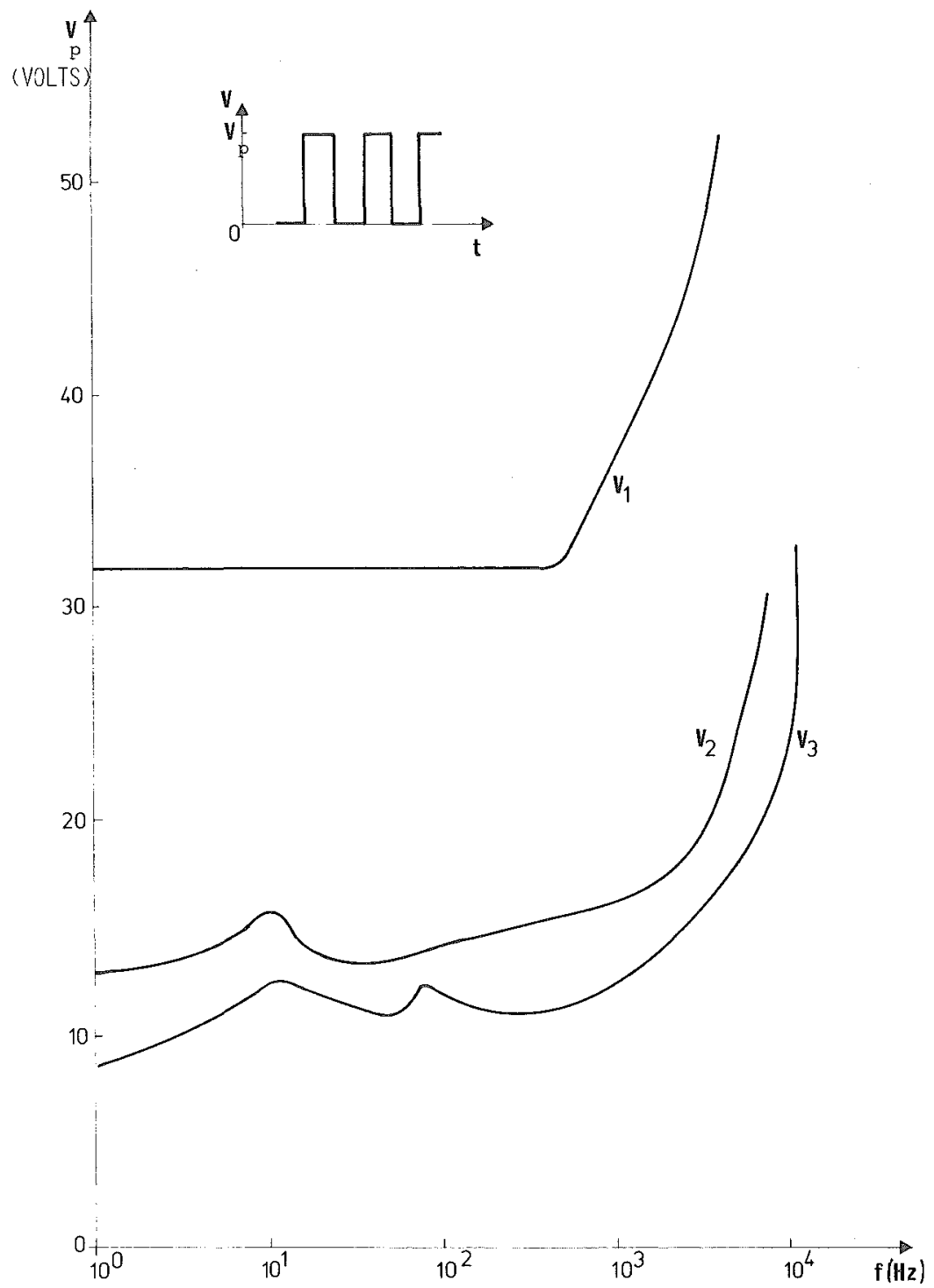

FIGURE 8 Stability chart of threshold voltage vs frequency for pulsed waves.

out the fact that the voltage $V_{3}$ for both signals is the same if the distance between top and bottom for the square voltage is taken.

We have studied this behavior with parallel (sandwiched) cells, but did not get any domain structure for frequencies higher than $300 \mathrm{~Hz}$ that 
corresponded to previous studies by other authors. We think the twisted geometry of the molecules is important and that it is possible with this configuration that the behavior will resemble that obtained with cholesteric liquid crystals. A more detailed study will be published by us shortly.

\section{CONCLUSIONS}

A new type of domain, in twisted-wedge nematic structures, is reported. These domains appear as a two-dimensional grating, having a structure similar to that of a Sigillaria trunk impression from the Late Carboniferous Period to be seen at the Natural Sciences Museum of the Smithsonian Institution (Washington, D.C.). These domains appear up to frequencies near $10 \mathrm{kHz}$, both for pulsed and square voltages. They appear over a voltage range larger than that for Williams domains.

\section{Acknowledgment}

This work was supported by the Spanish Comision Asesora de Investigación Cientifica y Técnica (Grant 3864-79).

\section{References}

1. P. G. de Gennes, The Physics of Liquid Crystals, Clarendon Press, Oxford (1974).

2. W. J. A. Goossens in Advances in Liquid Crystals, Vol. 3 (Ed. G. H. Brown), Academic Press, New York (1978).

3. Orsay Liquid Crystal Group, Phys. Lett., 39A, 181 (1972).

4. S. Chandrasekhar, Liquid Crystals, Cambridge University Press, Cambridge (1977).

5. P. Petrescu and M. Giurgea, Phys. Lett., 59A, 41 (1976).

6. J. A. Martín-Pereda and M. A. Muriel in Abstracts of the Ninth International Conference on Liquid Crystals, Bangalore, India, Dec. 6-10, 1982, paper E-14P.

7. M. A. Muriel and J. A. Martín-Pereda, in Proceedings of the 1980 European Conference on Optical Systems and Applications, Utrecht, Sept. 23-25, 1980, paper L2.

8. J. A. Martín-Pereda and M. A. Muriel, J. Opt. Soc. America, 70, 1610 (1980).

9. J. A. Martín-Pereda and M. A. Muriel, Opt. Lett, 5, 494, (1980).

10. W. Greubel and U. Wolff, Appl. Phys. Lett., 19, 213 (1971). 